\title{
Gestion des risques d'ambiguïté référentielle dans les textes juridiques : ledit et susdit
}

\author{
Marta Sobieszewska ${ }^{1}$ \\ Université Marie Curie-Sklodowska, Pl. M. Curie-Skłodowskiej 4A, 20-031 Lublin, \\ Pologne
}

Résumé. L'article vise à apporter quelques éléments de réflexion sur l'ambiguïté référentielle comme source potentielle des problèmes d'interprétation des textes juridiques. La prévention des risques de confusion référentielle est strictement liée au fonctionnement des formes de reprises spécialisées dans le contrôle de tels risques (cf. Charolles : 1995). L'analyse du fonctionnement des formes anaphoriques, telles que ledit $+\mathrm{N}$ et $(l e)+(\mathrm{N})+$ susdit, nous permet d'aborder les questions qui occupent toujours une place marginale dans les recherches linguistiques.

\begin{abstract}
Management of referential ambiguity risks in legal texts: said and aforesaid. The article aims to provide some elements of reflection on referential ambiguity as a potential source of problems in the interpretation of legal texts. The prevention of the risks of referential confusion is strictly linked to the functioning of the forms of takeovers specialized in the control of such risks (cf. Charolles: 1995). The analysis of the functioning of anaphoric forms, such as said $+\mathrm{N}$ and $($ the $)+(\mathrm{N})+$ aforesaid, allows us to propose a track to animate research on the questions which always occupy a marginal place in linguistic research.
\end{abstract}

\section{Introduction}

L'influence des activités des sémanticiens référentiels (cf., parmi d'autres, Charolles, Corblin, Kleiber) nous a amené à nous poser une question : quels sont les avantages induits par les reprises en ledit et susdit dans les textes juridiques? Dans une optique discursive et fonctionnelle, ces reprises cessent de paraître archaïques et marginales.

L'objectif de cet article est d'apporter quelques éléments de réflexion sur l'ambiguité référentielle comme source potentielle des problèmes d'interprétation des textes juridiques. L'analyse du fonctionnement des formes anaphoriques, telles que ledit $+\mathrm{N}$ et $(l e)+(\mathrm{N})+$ susdit, nous permet d'aborder les questions qui suscitent toujours peu d'intérêt chez les linguistes.

Le corpus étudié réunit 30 décisions rendues par la Cour de cassation entre 2011-2019 et publiées sur le site officiel de la Cour. Ce choix est dicté tout d'abord par le fait que la tradition érige les arrêts de la Cour de cassation en parangon de la clarté. Nul doute qu'un texte obscur et ambigu ne peut garantir l'efficacité du système juridique auprès des citoyens. En revanche, « un texte lisible est un texte intelligible, c'est-à-dire aisément compréhensible » (cf. Lasserre- Kiesow, 2002 : 1158). Il nous semble donc intéressant de considérer les textes qui se reconnaissent à leur clarté et à leur précision.

$\mathrm{Vu}$ le principe selon lequel l'Analyse du Discours se développe de façon interdisciplinaire, il nous semble justifié de proposer l'approche jurilinguistique (qui conjugue deux compétences diverses : linguistique et juridique). C'est ce que Charaudeau (2012) appelle une «interdisciplinarité focalisée ».

\footnotetext{
${ }^{1}$ marta.sobieszewska@poczta.umcs.lublin.pl
} 


\section{Ledit $+\mathbf{N}$ - anaphorique textuel par excellence}

Ledit n'a pas suscité, sauf de rares exceptions (quelques remarques dans les études de Raible (1972 : 57), Wilmet (1997 : 230) ; des articles : Lavric (2002, 2010), Whittaker (2003a, 2003b), Sobieszewska (2019)), l'intérêt des linguistes. Ce déterminant est composé de l'article défini le (la, les) contractable en $d u$ (de la, des) ou au (à la, aux) et du participe passé du verbe dire (dit, dite, dits, dites). D'après Wilmet (1997 : 230), il n’y a aucune raison de distraire ledit d'un ensemble, qu'il appelle " quantifiants-caractérisants déictiques 》explicitant ces deux composantes : l'article défini, contractable et le participe passé du verbe dire, qui cantonne le paradigme à l'anaphore. Nous appellerons anaphore une relation (asymétrique, selon Milner, 1982) de dépendance contextuelle entre une expression indexicale et son contexte antérieur, telle qu'elle permet de découvrir le référent de celle-ci.

Ledit (avec ses variantes ladite, lesdits, lesdites...) est un déterminant représentatif pour son « domaine d'origine ", c'est-à-dire les textes administratifs et juridiques (depuis le XIII ${ }^{\mathrm{e}}$ siècle), de même que pour les " genres sérieux » (De Wolf, 2003 : 343). L'examen des arrêts de la Cour de cassation permet de découvrir la fréquence élevée des SN comportant l'expression anaphorique ledit $+\mathrm{N}$.

Ledit $+\mathrm{N}$ apparait (presque) toujours dans la partie finale de l'arrêt (dispositif). Voyons quelques exemples :

$/ 1 /$.

CASSE ET ANNULE, en toutes ses dispositions, l'arrêt rendu le 13 février 2018, entre les parties, par la cour d'appel de Reims ; remet, en conséquence, la cause et les parties dans l'état où elles se trouvaient avant ledit arrêt et, pour être fait droit, les renvoie devant la cour d'appel de Nancy ; (Civ. $2^{\mathrm{e}}$, Arrêt nº63 du 16 mai 2019)

$12 /$.

CASSE ET ANNULE, en toutes ses dispositions, l'arrêt rendu le 28 février 2018, entre les parties, par la cour d'appel de Paris ; remet, en conséquence, la cause et les parties dans l'état où elles se trouvaient avant ledit arrêt et, pour être fait droit, les renvoie devant la cour d'appel de Versailles ; (Civ. $1^{\text {ère }}$, Audience publique du jeudi 19 septembre 2019, pourvoi ${ }^{\circ} 18-18433$ )

$/ 3 /$.

CASSE ET ANNULE, mais seulement en ce qu'il a rejeté les demandes de nullité et d'octroi de dommages-intérêts formées par M. X..., ès qualités, l'arrêt rendu le 19 mai 2010, entre les parties, par la cour d'appel de Paris ; remet, en conséquence, sur ces points, la cause et les parties dans l'état où elles se trouvaient avant ledit arrêt et, pour être fait droit, les renvoie devant la cour d'appel de Paris, autrement composée ; (Comm., Audience publique du mardi 4 octobre 2011, pourvoi $\mathrm{n}^{\circ} 10$ 20956)

Dans les exemples cités ci-dessus, ledit arrêt peut être aisément remplacé par cet arrêt, mais difficilement par l'arrêt, ce qui montre la faculté d'opérer un contraste interne entre les éléments (cf. F. Corblin, 1983). Grâce à la composante lexicale de « verbum dicendi », ledit a une valeur anaphorique plus forte que l'article défini. Il se pourrait que pour désambiguïser le texte, ledit prenne temporairement le relais du démonstratif. C'est ainsi que « ledit arrêt », celui dont on vient de parler, s'oppose clairement à " l'arrêt rendu le... + date » qui se trouve dans le même paragraphe. Le syntagme nominal contenant ledit reprend un référent qui joue un rôle de premier plan dans le texte. Par conséquent, l'arrêt le plus important est celui qui contient l'occurrence de ledit.

Mais s'il est vrai que ledit $+\mathrm{N}$ est susceptible d'être remplacé par un démonstratif, quelle est la raison de son existence ? Même si la fonctionnalité de ledit $+\mathrm{N}$ se rapproche de celle des démonstratifs, il y a une différence : ledit est incapable de marquer une référence déictique au sens situationnel. De plus, l'infidélité en reprise est beaucoup plus grande avec le démonstratif qu'avec ledit $+\mathrm{N}$.

Ledit $+\mathrm{N}$ ne se limite pas au dispositif de l'arrêt ; il apparaît également là où l'antécédent est difficile à reprendre par les anaphoriques classiques.

$14 /$.

Attendu que pour rejeter la demande, l'arrêt retient qu'en produisant les pièces demandées, la banque divulguerait les informations figurant au verso des chèques et porterait ainsi atteinte au secret dont sont titulaires les bénéficiaires desdits chèques ;

Qu'en se déterminant ainsi, sans rechercher si la communication à M. et Mme R... des informations figurant au verso des chèques qu'ils avaient émis n'était pas indispensable à l'exercice de leur droit à la preuve, pour rechercher l'éventuelle responsabilité de la banque lors de l'encaissement desdits chèques, et proportionnée aux intérêts antinomiques en présence, incluant la protection du secret dû aux bénéficiaires de ces chèques, la cour d'appel a privé sa décision de base légale ; (Comm., Arrêt n462 du 15 mai 2019) 
desdits chèques - des chèques (x2);

$15 /$.

CASSATION SANS RENVOI et cassation sur les pourvoi formés par :

- le procureur général près la cour d'appel de Douai,

- contre l'arrêt de la chambre de l'instruction de ladite cour d'appel, en date du 2 janvier 2019, qui, dans la procédure suivie contre M. F... H... des chefs d'infractions à la législation sur les stupéfiants et association de malfaiteurs en récidive, s'est déclarée incompétente pour statuer sur sa demande de mise en liberté;

- contre l'arrêt de ladite cour d'appel, 4e chambre, en date du 24 janvier 2019, qui, dans la même procédure, a annulé le jugement du tribunal correctionnel renvoyant les parties à mieux se pourvoir sur la demande de mise en liberté de M. H... et ordonné sa mise en liberté . (Crim., Audience publique du mardi 7 mai 2019)

ladite cour d'appel x 2 - la cour d'appel de Douai;

$/ 6 /$.

Mais attendu que, le droit au respect de la vie privée et le droit à la liberté d'expression ayant la même valeur normative, il appartient au juge saisi de rechercher un équilibre entre ces droits et, le cas échéant, de privilégier la solution la plus protectrice de l'intérêt le plus légitime ; que, pour effectuer cette mise en balance des droits en présence, il y a lieu de prendre en considération la contribution de la publication incriminée à un débat d'intérêt général, la notoriété de la personne visée, l'objet du reportage, le comportement antérieur de la personne concernée, le contenu, la forme et les répercussions de ladite publication, (...). (Civ. 1 ère, Arrêt n822 du 10 octobre 2019)

ladite publication - la publication ;

$/ 7 /$.

qu'en annulant la transcription des actes de naissance des enfants X... par cela seul qu'ils étaient nés en exécution d'une convention portant sur la gestation pour le compte d'autrui, la cour d'appel, qui a ainsi pénalisé ces enfants, en les privant de la nationalité de leurs parents, à raison de faits qui ne leur étaient pourtant pas imputables, a violé l'article 14 de la Convention européenne des droits de l'homme combiné avec l'article 8 de ladite Convention. (Assemblée plénière, Audience publique du vendredi 4 octobre 2019, pourvoi $\mathrm{n}^{\circ} 10-19053$ )

ladite Convention - la Convention européenne des droits de l’homme;

$18 /$.

Mais attendu qu'en statuant ainsi, alors qu'il résulte de ses propres constatations que les propos, par lesquels le prévenu se prévalait de son appartenance personnelle à une organisation terroriste, responsable de plusieurs attentats commis dans une période récente sur le sol français, pour intimider et menacer ses interlocuteurs, ne pouvaient, compte tenu des circonstances dans lesquelles ils avaient été tenus, que susciter en eux des sentiments de crainte et de rejet, exclusifs de tout regard favorable sur ladite organisation, la cour d'appel a méconnu le sens et la portée du texte susvisé et du principe ci-dessus rappelé ; (Crim., Arrêt n928 du 4 juin 2019)

ladite organisation - une organisation terroriste ;

$19 /$.

Attendu, selon l'arrêt attaqué (Pau, 17 octobre 2016), qu'après avoir été omise, en 1994 et 2013, du tableau de l'ordre des avocats au barreau de Bayonne, Mme Y... a, en février 2015, sollicité sa réinscription ; que, par délibération du 13 mai suivant, le conseil de l'ordre dudit barreau (le conseil de l'ordre) a rejeté cette demande ; (Civ. 1 ${ }^{\text {ère }}$, Audience publique du mercredi 14 février 2018, pourvoi $\mathrm{n}^{\circ}$ 16-27909)

dudit barreau - le barreau de Bayonne ;

Le propre de l'anaphore par ledit $+\mathrm{N}$ est de reprendre des antécédents qui remontent trop loin pour un anaphorique «normal » (cf. Lavric, 2010 : 143). De plus, là où l'antécédent est un simple nom, il est repris à l'identique : des chèques - desdits chèques (ex. 4) ; la publication - ladite publication (ex. 6), pourtant là où l'antécédent est accompagné de mots ou de groupes de mots, il les perd dans la répétition : la cour d'appel de Douai

- ladite cour d'appel (ex. 5), la Convention européenne des droits de l'homme - ladite Convention (ex. 7), une organisation terroriste - ladite organisation (ex. 8), le barreau de Bayonne - dudit barreau (ex. 9). Ainsi le syntagme anaphorique devient-il un hyperonyme sémantique ou bien la reprise «identique hyperonymique »

( cf. E. Lavric, $2010: 143$ ). 
Il est évident que la reprise d'un antécédent éloigné est le propre de l'anaphorique ledit. Mais il se peut aussi que ledit $+\mathrm{N}$ ait plusieurs reprises : il s'agit de la $\mathrm{n}^{\text {ième }}$ mention du même antécédent qui se produit sous forme de reprise identique. Observons :

$/ 10 /$.

1) L'article 2, sous b), de la directive 93/13/CEE du Conseil, du 5 avril 1993, concernant les clauses abusives dans les contrats conclus avec les consommateurs, doit être interprété en ce sens que le salarié d'une entreprise et son conjoint, qui concluent avec cette entreprise un contrat de crédit, réservé, à titre principal, aux membres du personnel de ladite entreprise, destiné à financer l'acquisition d'un bien immobilier à des fins privées, doivent être considérés comme des " consommateurs ", au sens de cette disposition ;

2) L'article 2, sous c), de la directive doit être interprété en ce sens que ladite entreprise doit être considérée comme un "professionnel », au sens de cette disposition, lorsqu'elle conclut un tel contrat de crédit dans le cadre de son activité professionnelle, même si consentir des crédits ne constitue pas son activité principale ; (...)

Par arrêt du 19 mars 2019 (C-590/17), la CJUE a dit pour droit que l'article 2, sous b) et sous c) de la directive 93/13/CEE du Conseil du 5 avril 1993, concernant les clauses abusives dans les contrats conclus avec les consommateurs, doit être interprété en ce sens que le salarié d'une entreprise et son conjoint, qui concluent avec cette entreprise un contrat de crédit, réservé, à titre principal, aux membres du personnel, et destiné à financer l'acquisition d'un bien immobilier à des fins privées, doivent être considérés comme des « consommateurs » et que ladite entreprise doit être considérée comme un «professionnel », lorsqu'elle conclut un tel contrat de crédit dans le cadre de son activité professionnelle, même si consentir des crédits ne constitue pas son activité principale. (Civ. $1^{\text {ère }}$, Audience publique du mercredi 5 juin 2019, pourvoi n 16-12519)

Dans cet exemple, ledit $+\mathrm{N}$ se restreint à l'anaphore fidèle (identique) une entreprise/cette entreprise où le $\mathrm{N}$ est le même que le nom tête de l'antécédent.

Ledit + N peut également faire partie des chaînes de référence (C. Schnedecker : 1997) qui se rapportent soit aux référents non-humains :

$/ 11 /$.

Aux motifs que "selon le contrat souscrit, les consorts Y...-X... sont bénéficiaires de la somme assurée en cas de décès de $M$. Z ... qui est censé à titre principal récupérer son investissement à l'issue d'une durée de 30 ans ; que ledit contrat prévoit expressément la possibilité d'un rachat total ou partiel ; (...) que le rachat étant admis, il devient sans intérêt d'examiner la responsabilité contractuelle de l'assureur retenue par le tribunal dont la décision conduisait pourtant à condamner l'assureur tout en laissant subsister le contrat" (arrêt attaqué, p. 4, § 2 à 6) (Mix., Arrêt n 262 du 22 février 2008).

- le contrat (le premier maillon de la chaîne de référence) - ledit contrat (anaphore par ledit $+\mathrm{N}$ ) - le contrat (reprise à l'identique de l'antécédent) ;

soit aux référents humains :

$/ 12 /$.

Sauf disposition contraire de l'article 16 du règlement d'application, lorsqu'une personne exerce son activité dans un État membre autre que l'État membre compétent conformément au titre II du règlement de base, l'employeur ou, si la personne n'exerce pas une activité salariée, la personne concernée en informe, préalablement lorsque c'est possible, l'institution compétente de l'État membre dont la législation est applicable. Cette institution remet à la personne concernée l'attestation visée à l'article 19 , paragraphe 2 , du règlement d'exécution et met sans délai à la disposition de l'institution désignée par l'autorité compétente de l'État membre où l'activité est exercée des informations sur la législation applicable à ladite personne, conformément à l'article 11 , paragraphe 3 , point b), ou à l'article 12 du règlement de base". (Crim., 8 janvier 2019, pourvoi nº 17-82553).

L'ouverture de la chaîne de référence se fait ici par le premier maillon (une personne) qui est repris par les anaphores fidèles (la personne $\mathrm{x} 3$ ), puis par ledit $+\mathrm{N}$ (ladite personne), qui, à son tour, prend sa place dans la suite de la chaîne de référence.

Il va de soi que les chaînes de référence dans les textes juridiques ont leurs propres caractéristiques qui attendent leur mise en lumière. Les textes non-narratifs comportent en eux-mêmes des traits discursifs d'ordre pragmatique qui implique (et explique, aussi) que les discours dont ils sont porteurs soient intégré à une tradition orale. C'est, d'une manière générale, le cas des textes juridiques, qui sont, en majorité, prononcés à l'oral (toute décision de justice doit être prononcée devant un auditoire) (Sobieszewska, 2019:81). 
Une autre spécificité de ledit $+\mathrm{N}$ réside dans le fait que l'identification de la source d'interprétation se fait par le biais du texte et non par le biais de la mémoire discursive, à la différence des autres expressions anaphoriques (cf. Whittaker : 2003).

$/ 13 /$.

L'article 2, sous b) et d), de la directive 95/46/CE doit être interprété en ce sens que, d'une part, l'activité d'un moteur de recherche consistant à trouver des informations publiées ou placées sur Internet par des tiers, à les indexer de manière automatique, à les stocker temporairement et, enfin, à les mettre à la disposition des internautes selon un ordre de préférence donné doit être qualifiée de « traitement de données à caractère personnel », au sens de cet article 2, sous b), lorsque ces informations contiennent des données à caractère personnel et, d'autre part, l'exploitant de ce moteur de recherche doit être considéré comme le « responsable » dudit traitement, au sens dudit article 2, sous d) ; (Civ. 1 ${ }^{\text {ère }}$, Audience publique du mercredi 14 février 2018, pourvoi n 17-10499)

$/ 14 /$.

Viole également les textes susvisés, la cour d'appel qui exclut le caractère abusif d'une clause de résiliation de plein droit du contrat de prêt en cas de rupture du contrat de travail, au motif qu'elle s'inscrit dans un contrat qui présente des avantages pour le salarié et équilibre ainsi ladite clause, alors qu'est abusive la clause de résiliation stipulant la résiliation de plein droit du contrat de prêt pour une cause extérieure à ce contrat (...); (Civ. 1 ${ }^{\text {ère }}$, Audience publique du mercredi 5 juin 2019, pourvoi $\left.\mathrm{n}^{\circ} 16-12519\right)$

Il est à noter que les textes juridiques créent un environnement favorable à cet anaphorique. Cependant, l'emploi récurrente des séquences ledit + N n'est pas uniquement une question de style : il est essentiel pour la cohésion du texte.

$/ 15 /$.

Il est fait grief au jugement attaqué d'avoir condamné la société THOMAS COOK à régler aux époux X... la somme de 641,16 euros, avec intérêts de droit ; AUX MOTIFS QUE « sur l'application $d u$ règlement communautaire $n^{\circ} 261 / 2004$, les requérants font valoir que ledit règlement $\mathrm{n}$ 'est pas seulement applicable au transporteur mais aussi à l'organisateur de voyages ; qu'ils se fondent pour ce faire sur l'article 3 qui stipule que le paragraphe 1 s'applique à condition que les passagers « aient été transférés par le transporteur aérien ou l'organisateur de voyages du vol pour lequel ils possédaient une réservation vers un autre vol, quelle qu'en soit la raison »; que rien ne permet d'exclure en l'espèce l'application $d u$ présent règlement; qu'en effet au paragraphe 3 dudit document il est indiqué : " le présent règlement ne s'applique pas aux passagers qui voyagent gratuitement ou à un tarif réduit non directement ou indirectement accessible au public. (...)

LORS QUE le règlement communautaire $n^{\circ}$ 261/2004 du 11 février 2004, «établissant des règles communes en matière d'indemnisation et d'assistance des passagers en cas de refus d'embarquement et d'annulation ou de retard important d'un vol », désigne le « transporteur aérien effectif » comme débiteur exclusif des obligations d'assistance et d'indemnisation qu'il édicte ; qu'en décidant cependant en l'espèce que ledit règlement n'était pas seulement applicable au transporteur aérien mais aussi à l'organisateur du voyage, la Juridiction de proximité a violé, par fausse application, les articles 3 et 9 de ce texte. Moyen produit au pourvoi incident par Me Le Prado, avocat aux Conseils pour les époux X........)

AUX MOTIFS QUE les époux X... font valoir qu'ils ont été contraints de se rendre à l'aéroport d'ORLY, plutôt qu'à celui de ROISSY pour intercepter le vol à destination de l'EGYPTE et d'acquérir à cette fin, de nouveaux titres de transports et de nouvelles nuitées à proximité d'ORLY ; mais que l'article 25 des conditions générales de vente de la société défenderesse stipule : « les préacheminements et les post acheminements pris à la seule initiative du client relèvent en tout état de cause de sa responsabilité exclusive »; qu'il est mentionné sur le bulletin d'inscription versé aux débats que les conditions générales de vente figurent au verso dudit document ceci dans le respect des dispositions légales ; que les époux X... se contentent de procéder par affirmation en soutenant qu'ils n'ont pas eu connaissance desdites conditions générales de vente ; (...).

que dès lors, en retenant, pour affirmer que les époux X... avaient eu connaissance des conditions générales de vente de la société THOMAS COOK, et en particulier de l'article 25 de ces conditions générales de vente, qu'il était mentionné sur le bulletin d'inscription versé aux débats que les conditions générales de vente figuraient au verso dudit document, de sorte que les époux X... se contentaient de procéder par affirmation en soutenant qu'ils n'avaient pas eu connaissance desdites conditions générales de vente, l'existence de telles conditions étant rappelée de façon explicite sur le bulletin d'inscription signé par les requérants, la juridiction de proximité a dénaturé le bulletin d'inscription et les conditions générales de vente y annexées, en violation de l'article 1134 du Code civil ; (Civ. $1^{\text {ère }}$, Audience publique du jeudi 8 mars 2012, pourvoi n ${ }^{\circ} 11-10226$ ) 
ledit règlement - le règlement communautaire $n^{\circ}$ 261/2004 (anaphore infidèle);

dudit document - le bulletin d'inscription (anaphore infidèle);

desdites conditions générales de vente - des conditions générales de vente (anaphore fidèle);

dudit document - le bulletin d'inscription (anaphore infidèle);

desdites conditions générales de vente - les conditions générales de vente (anaphore fidèle).

Tout phénomène de deuxième apparition qui, par mémorisation, est lié à une première apparition dans le contexte est pour nous une anaphore et, quand le nom de la deuxième apparition est le même que celui de la première apparition, nous parlons d'anaphore fidèle. En revanche, l'anaphore infidèle est entendue ici comme reprise par hyperonyme : la tête est différente de celle de l'antécédent, mais N1 et N2 évoquent tous deux le « même objet de pensée » (Blanche- Benveniste \& Chervel, 1966 : 29-31).

Le survol des exemples présentés ci-dessus permet d'observer que, dans la majorité des occurrences, il s'agit d'une anaphore lexématique fidèle où le $\mathrm{N}$ est le même que le nom tête de l'antécédent. L'antécédent peut être accompagné d'épithète ou de complément, c'est ainsi que le syntagme anaphorique devient un hyperonyme des mots désignés par l'antécédent. En effet, ledit $+\mathrm{N}$ peut reprendre un antécédent long et complexe, ce qui permet, d'une part, d'exercer le contrôle des risques d'ambiguité et, de l'autre, d'assurer la cohérence nécessaire pour l'identification du référent.

Bref, ledit $+\mathrm{N}$ est un anaphorique textuel particulièrement spécialisé dans la reprise d'un antécédent éloigné dans l'espace textuel. Étant donné que la portée sémantique des déterminants démonstratifs n'est toujours pas suffisante, il apparaît comme un déterminant qui permet de résoudre les références ambiguës. Grâce à l'emploi de l'expression ledit

+ N, la distance (même importante) entre l'expression anaphorique et son antécédent n'est plus un obstacle ni pour la clarté, ni pour la précision du message. Par contre, l'assistance des expressions anaphoriques pronominales $(i l$, elle, le, la , les... etc.) impliquent habituellement que l'antécédent se trouve déjà dans la mémoire immédiate de l'interlocuteur et qu'il est clair pour lui à quoi le pronom se réfère.

Enfin, pour s'assurer qu'il s'agit bien des formes reservées à un usage juridique, il suffit de vérifier, en allant voir sur la base de donnés Frantexte. Les exemples trouvés sont très peu nombreux et ils datent du XIXe siècle. Ils font tous référence au cadre juridique :

$/ 16 /$.

Monsieur Fix, répondit le consul, vous parlez d'une telle façon que je vous souhaiter vivement de réussir ; (..).

Monsieur le consul, répondit dogmatiquement l'inspecteur de police, les grands voleurs ressemblent toujours à d'honnêtes gens. Vous comprenez bien que ceux qui ont des figures de coquins n'ont qu'un parti à prendre, c'est de rester probes, sans cela ils se feraient arrêter. Les physionomies honnêtes, ce sont celles-là qu'il faut dévisager surtout. Travail difficile, j'en conviens, et qui n'est plus du métier, mais de l'art. "

On voit que ledit Fix ne manquait pas d'une certaine dose d'amour-propre. M223 | VERNE Jules - Le Tour du monde en quatre-vingts jours (1873) (p. 26).

Ledit + nom propre (Fix) ;

$/ 17 /$.

Attendu, reprit le juge, attendu que la loi anglaise entend protéger également et rigorieusement toutes les religions des populations de l'Inde, le délit étant avoué par le sieur Passepartout convencu d'avoir violé d'un pied sacrilège le pavé de la pagode de Malebar-Hill, à Bombay,dans la journée du 20 octobre, condamne ledit Passepartout à quinze jours de prison et à une amende de trois cents livres (7500 fr.). M223 |VERNE Jules- Le Tour du monde en quatre-vingts jours (1873) (p. 81).

ledit + nom propre (Passepartout)

$/ 18 /$.

47. Tout acte de l'état civil des Français et des étrangers, fait en pays étranger, fera foi, s'il a été rédigé dans les formes usitées dans ledit pays. Code civil des Français (1804) L 1, DES PERSONNES (p. 11).

ledit pays - pays étranger ;

$/ 19 /$.

Quiconque réclamera un droit échu à un individu dont l' existence ne sera pas reconnue, devra

prouver que ledit individu existait quand le droit a été ouvert : jusqu' à cette preuve, il sera déclaré 
non ecevable dans sa demande. Code civil des Français, (1804), DES PERSONNES, (p. 28).

\section{ledit individu - un individu}

Ledit est un archaïsme qui n'est pratiquement jamais employé dans d'autres genres d'écrit. Il se remarque (« saille ») d'autant plus qu'il a perdu tout contact avec la langue parlée. Il possède de ce fait un caractère particulier de rareté et de solennité.

\section{3. (Le) $+(\mathrm{N})+$ susdit $-\mathrm{SN}$ anaphorique démonstratif hybride}

Susdit n'appartient qu'à la nomenclature des termes du Palais ; c'est probablement une des raisons pour laquelle il n'a jamais vraiment suscité l'intérêt des linguistes.

C'est un anaphorique ayant pour fonction de permettre le repérage d'un référent. Il possède des marqueurs référentiels hybrides (à la fois déictiques et anaphoriques), ce qui oppose le déictique -sus, à l'anaphorique dit. Le préfixe sus- renvoie explicitement à ce dont il a été question précédemment : il dirige le regard vers le haut et indique le

« lieu de résidence » du référent. C'est une action strictement localisante. Pendant que la composante de « verbum dicendi » (dit), qui possède le statut métalinguistique spécial, signale explicitement qu'il est question du référent repris par le $+\mathrm{N}+$ susdit. Observons :

$120 /$.

ET, AUX MOTIFS ADOPTES DES PREMIERS JUGES, QUE la faute commise par le conducteur du véhicule terrestre à moteur a, conformément à l'article 4 de la loi du 5 juillet 1985, pour effet de limiter ou d'exclure l'indemnisation des dommages qu'il a subis ; (...) qu'ainsi, l'irrespect du Code de la route par Henry X..., qui n'a pas marqué le temps d'arrêt imposé par le panneau stop, s'est engagé sur la route de Perrin alors que l'autobus conduit par Alphonse Y... y circulait, est une faute, laquelle est en outre la cause exclusive de l'accident; que cette faute ne peut emporter, au sens de l'article 4 susdit, qu'exclusion de leur indemnisation ; (Civ. 2 ${ }^{\mathrm{e}}$, Audience publique du 16 juin 2011, pourvoi $\left.\mathrm{n}^{\circ} 10-18075\right)$

l'article 4 susdit - l'article mentionné ci-haut, celui de la loi du 5 juillet 1985 ;

$121 /$.

Attendu qu'il résulte de l'arrêt attaqué et des pièces de procédure que la société civile immobilière Les Galmouches est, depuis 2005, propriétaire d'un bien sur la commune de Joinville ; que cet immeuble, primitivement donné en location, a presqu'immédiatement été libre de toute occupation et qu'ultérieurement, il a été déclaré par l'administration compétente en état d'abandon manifeste ; que dans la soirée du 3 août 2013, vers 20h, deux mineurs, C... Y... et Benoît X... ont pénétré dans cet immeuble, y ont mis le feu à divers objets avant de quitter les lieux ; qu'un incendie a pris naissance dans le bâtiment et s'est propagé à sept maisons, dont six ont été détruites, notamment celle qui appartenait à la société civile immobilière susdite ; (Crim., Arrêt n³425 du 15 janvier 2019)

la société civile immobilière susdite - la société civile immobilière Les Galmouches ;

$122 /$.

qu'enfin, le décret 2012-639 du 4 mai 2012, entré en vigueur le 1er juillet 2012, applicable aux opérations pour lesquelles le dossier de consultation relatif au marché a été publié à compter de cette date (cf article

5) codifié aux articles R. 4412-94 à R. 4412-143 nouveaux du code du travail, qui précise les modalités selon lesquelles la protection des travailleurs contre les risques d'exposition à l'amiante est assurée, notamment en ce qui concerne la détermination delà valeur limite d'exposition professionnelle, les conditions du contrôle du respect de cette valeur limite ainsi que les modalités de mesurage des empoussièrements, qui fixe les règles techniques, les moyens de prévention collective et les types d'équipements individuels nécessaires à la protection des travailleurs contre ces expositions, qui prévoit aussi un dispositif unique de certification des entreprises d'encapsulage ou de retraite de matériaux contenant de l'amiante, n'est pas applicable aux faits de l'espèce, en considération de la date du chantier dont le dossier de consultation est antérieur au 1er juillet 2012, puisque le marché passé entre la société Mandevilla et la société Vinci construction terrassement a été signé le 21 mai 2012, avec une date de commencement des travaux fixée au 25 mai 2012 ; que toutefois, le décret 2006-76 du 30 juin 2006 relatif à la protection des travailleurs contre les risques liés à l'inhalation de poussières d'amiante n'a toutefois pas été abrogé par le décret susdit. (Crim.,Audience publique du mercredi 19 avril 2017, pourvoi $\mathrm{n}^{\circ}$ 16-80695) 
décret susdit est la reprise anaphorique du décret 2012-639 du 4 mai 2012.

$L e+\mathrm{N}+$ susdit connaît aussi des emplois typiques de reprise à l'identique de l'antécédent :

$123 /$.

que l'arrêté du 4 mai 2007 relatif à la mesure de la concentration des fibres d'amiante sur les lieux de travail et aux conditions d'accréditation des laboratoires a ainsi été pris et publié le 16 mai 2007 ; qu'il prévoit notamment en son article 2 que l'accréditation est délivrée par le COFRAC ou tout organisme signataire de l'accord européen ; que, pour respecter les obligations communautaires issues de la directive 2009/ 148/ CE tout en adaptant le dispositif de métrologie aux dispositions issues du décret 2012-639 du 4 mai 2012 relatif aux risques d'exposition à l'amiante, cet arrêté a été abrogé par l'arrêté du 14 août 2012, sauf en ce qui concerne l'article 2 susdit, dont l'entrée en vigueur a été différée au 1er juillet 2013 ; (Crim.,Audience publique du mercredi 19 avril 2017, pourvoi n ${ }^{\circ}$ 16-80695)

Dans /16/., il s'agit de l'article 2 précédemment mentionné. La répétition du syntagme nominal + susdit (l'article 2 susdit) témoigne d'un souci de précision explicite dans le texte.

L'expression anaphorique le $+\mathrm{N}+$ susdit reprend souvent un groupe nominal ou un segment qui apparait clairement dans la partie précédente :

$124 /$.

« Statuant sur le pourvoi formé par :

- M. Y X,

contre l'arrêt de la chambre de l'instruction de la cour d'appel d'AIX-EN-PROVENCE, en date du 4 mars 2019, qui dans la procédure suivie contre lui des chefs de recel de biens provenant de vols en bande organisée, infractions à la législation sur les armes, le tout en récidive, et participation à une association de malfaiteurs en vue de la préparation d'un délit, a confirmé l'ordonnance du juge des libertés et de la détention rejetant sa demande de mise en liberté ; » (...)

Attendu qu'il résulte de l'arrêt attaqué et des pièces de procédure que M. O... a été mis en examen par un juge d'instruction des chefs susdits, et placé en détention provisoire par ordonnance du 25 octobre 2018 ; (Crim. Arrêt n 19-81971, 28 mai 2019)

des chefs susdits - des chefs de recel de biens ;

$125 /$.

Et aux motifs adoptés des premiers juges que par un document dénommé "valeur locative et conditions particulières » en date du 7 février 2006, les parties ont exposé désirer l'établissement entre elles d'un bail à long terme de 25 ans dressé par Maître C..., notaire, aux conditions exposées dans le document susdit, avec effet rétroactif au 01/01/06 ; (Civ. 3 ${ }^{\mathrm{e}}$, Arrêt $\mathrm{n}^{\circ}$ 16-10225n 17 mai 2018)

le document susdit - un document dénommé « valeur locative et conditions particulières »;

Comme dans le cas de ledit, susdit a une valeur anaphorique plus forte que l'article défini:

$126 /$.

Attendu qu'il résulte de l'arrêt attaqué et des pièces de procédure que la cour d'appel d'Aix-enProvence, par arrêt du 14 avril 2009, a déclaré les prévenus coupables d'infractions au code de l'urbanisme et les a condamnés à une amende, en ordonnant la remise en état des lieux sous astreinte ; que la société civile immobilière dirigée par l'un d'eux et propriétaire des lieux, ainsi que la société commerciale dirigée par l'autre et utilisatrice desdits locaux, ont été placées en liquidation judiciaire ; qu'au motif que le liquidateur judiciaire n'exécutait pas les travaux de remise en état imposés par l'arrêt susdit, les prévenus ont requis de la cour d'appel une dispense de paiement de l'astreinte ; (Crim., Audience publique du mardi 8 mars 2016, pourvoi n ${ }^{\circ} 5-82513$ )

Ici, l'arrêt susdit (c'est-à-dire arrêt du 14 avril 2009) peut être remplacé par cet arrêt, mais difficilement par l'arrêt. Le

$+\mathrm{N}+$ susdit est (presque toujours) susceptible d'être remplacé par un démonstratif, sans que le sens ou la référence de la phrase en soient modifiés.

Enfin, le $+\mathrm{N}+$ susdit peut être métatextuel et renvoyer explicitement à un autre texte :

$127 /$.

qu'elle ne mentionne pas davantage dans sa télécopie du 18 janvier 2017 ; qu'il n'est pas établi ni même allégué qu'elle ait eu la possession d'état d'enfant de Monsieur R... X...; que la réalité d'une possession d'état est en toute hypothèse démentie par plusieurs attestations, dont les auteurs relatent que Monsieur R... X... vivait seul ; qu'il ressort en outre d'un dossier médical que R... X... avait dû 
être hospitalisé en psychiatrie dans sa jeunesse pour un ancien éthylisme ; qu'il ressort encore des attestations susdites qu'il en avait conservé des séquelles qui le rendaient vulnérable, faible et influençable ; (Civ. $1^{\text {ère }}$, Audience publique du mercredi 15 mai 2019, pourvoi n ${ }^{\circ} 18-12602$ )

- celui des attestations des témoins.

Bref, le recours à la reprise par le $+\mathrm{N}+$ susdit ne semble pertinent que lorsque l'on a le sentiment que le locuteur n'avait pas d'autre choix. Bien que cette expression n'apparaisse (presque) jamais dans la langue générale, elle a une grande importance dans la langue juridique, où elle opère pour la cohésion textuelle.

\section{Conclusion}

L'analyse des expressions : ledit $+\mathrm{N}-\mathrm{SN}$ anaphorique et $(L e)+\mathrm{N}+$ susdit $-\mathrm{SN}$ anaphorique démonstratif hybride montre qu'elles sont toutes spécialisées dans la gestion des risques d'ambiguité référentielle. Prêtes, à chaque moment donné du discours, à reprendre un terme déjà cité (la distance, même considérable, entre l'expression anaphorique et son antécédent n'est pas un obstacle), ces expressions contribuent de façon efficace à la clarté et à la précision du message. Or, les procédés référentiels potentiellement ambigus sont plutôt rares dans les textes juridiques.

Ce donc, « Le fait que les grammaires ne consacrent qu'une place très minime aux expressions anaphoriques spécialisées dans le contrôle des risques d'ambiguiité référentielle est révélateur du peu d'intérêt qu'on leur porte en général. » (M. Charolles, 1995 : 90), qui nous a poussée à cette étude.

\section{Références bibliographiques}

Blanche-Benveniste, C., Chervel, A. (1966). « Recherches sur le syntagme substantif », Cahiers de lexicologie 9, p. 3-37.

Charaudeau, P. (2012). «Pour une interdisciplinarité focalisée. Réponses aux réactions », Revue Questions de

communication, $\mathrm{n}^{\circ} 21$,

pp. 171-206.

Charolles, M. (1995). « Comment repêcher les derniers ? Analyse des expressions anaphoriques en "ce dernier" »,

Pratiques : linguistique, littérature, didactique, Centre de recherche sur les médiations, Université de Lorraine, pp. 89-

Corblin, F. (1990). «Les groupes nominaux sans nom du français », (éds) Kleiber, G. et Tyvaert J.-E., pp. 63-80. Corblin,

F. (1995). Les formes de reprise dans le discours, Presses Universitaires de Rennes.

Corblin, F. (1998). «"Celui-ci" anaphorique : un mentionnel » Langue française, $\mathrm{n}^{\circ} 120$, Les démonstratifs : théorie linguistique et textes littéraires, sous la dir. de M.-N. Gary-Prieur et M. Léonard, pp. 33-43.

Corblin, F. (1999). «Les références mentionnelles : le premier, le dernier, celui-ci », A. Mettouch, H. Quintin. Mettouchi A et Quintin H., La référence (2), PUR Rennes, pp.107-123.

Kleiber, G. (1981). Problèmes de référence : description définies et noms propres, Paris, Klincksieck.

Kleiber, G. (1986). « Déictiques, embrayeurs, "token-réflexives", symboles indexicaux, etc. : comment les définir ?»,

L'Information Grammaticale, $\mathrm{n}^{\circ}$ 30, pp. 3-22.

Kleiber, G., Schnedecker, C., Tyvaert, J.-E. (1997). La continuité référentielle, Mets, Klincksieck.

Lasserre-Kiesow, V. (2002). «La compréhensibilité des lois à l'aube du XX siècle », Recueil Dalloz, pp. 1157-1160.

Lavric, E. (2002). «Locutions déterminatives phoriques textuelles : ce dernier, ce même, ledit, lequel, le ... en question, le... suivant (et leurs équivalents espagnols et allemands) ", Marek Kęsik (éd.): Référence discursive dans les langues romanes et slaves, Actes du colloque international de linguistique textuelle, Lublin, Wydawnictwo Uniwersytetu Marii Curie-Skłodowskiej, pp. 153-175.

Lavric, E. (2010). « Différents types de flèches : la phoricité dans tous ses états », Wenn Deiktika nicht zeigen: zeigende und nichtzeigende Funktionen deiktischer Formen in den romanischen Sprachen, Romanistische linguistik, Berlin, pp. 139-160.

Maillard, M. (1974). «Essai de typologie des substituts diaphoriques », Langue française, $\mathrm{n}^{\circ} 24$. 
Milner, J.-C. (1982). Ordres et raisons de langue, Paris, Seuil.

Raible, W. (1972). Satz und Text. Untersuchungen zu vier romanischen Sprachen, Tübingen (Niemeyer) (Beihefte zur Zeitschrift für romanische Philologie, Band 132).

Schnedecker, C. (1997). Nom propre et chaînes de références, Paris, Klincksieck.

Sobieszewska, M. (2019). Clarté et précision du discours juridique: procédés référentiels dans les arrêts de la Cour de cassation, Lublin, Wydawnictwo Uniwersytetu Marii Curie-Skłodowskiej.

Whittaker, S. (2003a). «Étude contrastive des expressions anaphoriques Ledit $N$ et Le $N$ en question », Revue de linguistique romane, 267-268, 67, 2003a, pp. 445-462.

Whittaker, S. (2003b). « Pour une description textuelle et discursive de l'expression anaphorique Ledit N», Journal of French Language Studies 13, 2 pp., pp. 159-176.

Wilmet, M. (1997). Grammaire critique du français, Hachette Supérieur, Duculot Louvain.

Wolf (de), A. (2003). «Un nouveau déterminant : le déterminant anaphorique - dit en français médiéval », Verbum 25/3, pp. 335-351. 